\title{
Artificial intelligence and machine learning: an introduction for orthopaedic surgeons
}

\author{
R. Kyle Martin ${ }^{1}$ D $\cdot$ Christophe Ley ${ }^{2} \cdot$ Ayoosh Pareek $^{3} \cdot$ Andreas Groll $^{4} \cdot$ Thomas Tischer $^{5} \cdot$ Romain Seil $^{6}$
}

Received: 2 August 2021 / Accepted: 2 September 2021 / Published online: 15 September 2021

(c) European Society of Sports Traumatology, Knee Surgery, Arthroscopy (ESSKA) 2021

\begin{abstract}
The application of artificial intelligence (AI) and machine learning to the field of orthopaedic surgery is rapidly increasing. While this represents an important step in the advancement of our specialty, the concept of AI is rich with statistical jargon and techniques unfamiliar to many clinicians. This knowledge gap may limit the impact and potential of these novel techniques. We aim to narrow this gap in a way that is accessible for all orthopaedic surgeons. With this manuscript, we introduce the concept of AI and machine learning and give examples of how it can impact clinical practice and patient care. Level of evidence VI.
\end{abstract}

Keywords Machine learning $\cdot$ Artificial intelligence $\cdot$ Orthopaedic surgery $\cdot$ Sports medicine

\section{Introduction}

Artificial intelligence (AI) and machine learning (ML) are said to be part of the next industrial revolution and represent a novel approach to health-related research and clinical care. The use of both AI and ML has been widespread throughout society for decades, but the introduction to patient care has been more gradual. Even within medicine there are discrepancies regarding the application of AI, with data-heavy fields like radiology and oncology leading the way. As a specialty, orthopaedic surgery has been a particularly late adopter of the tools and techniques that comprise AI [2, 3].

R. Kyle Martin

rkylemmartin@gmail.com

1 Department of Orthopedic Surgery, University of Minnesota, Minneapolis, MN, USA

2 Department of Applied Mathematics, Computer Science and Statistics, Ghent University, Ghent, Belgium

3 Department of Orthopaedic Surgery, Mayo Clinic, Rochester, MN, USA

4 Department of Statistics, TU Dortmund University, Dortmund, Germany

5 Department of Orthopaedic Surgery, University Medicine Rostock, Rostock, Germany

6 Department of Orthopaedic Surgery, Centre Hospitalier Luxembourg and Luxembourg Institute of Health, Luxembourg, Luxembourg
The incorporation of AI into the orthopaedic literature has only recently begun to increase. One explanation for this is a simple lack of understanding on the part of orthopaedic surgeons and researchers, who have been jokingly compared with the ox in terms of both strength and intelligence [17]. For clinicians, the understanding of AI principles, techniques, and clinical application is often difficult.

While there are many user guides [8] and technical descriptions [5] invaluable for in-depth understanding, there remains a need for a concise starting point for orthopaedic surgeons new to AI and ML. The goal of this editorial is to synthesize what can be a complex and developing field of orthopaedic research and simplify it for the average reader new to this field.

\section{The art of medicine}

Every day, clinicians are tasked with making informed decisions regarding diagnosis, appropriate treatment, and prognosis. These clinicians rely on pattern recognition, their understanding of the applicable literature, and their own training and experience to guide these decisions. For example, when the 16-year-old athlete presents with knee pain, we combine the information from the history, physical examination, and imaging to arise at the diagnosis of an anterior cruciate ligament (ACL) rupture. Both highquality rehabilitation and surgery are reasonable treatment 
options [1], and available evidence and surgeon experience can guide the decision-making discussion with the patient. Prognosis of both surgery and rehabilitation can be quoted as a "failure rate", also based on understanding of the existing literature and clinical experience of the surgeon. This gestalt has been coined the "art of medicine" since the clinical decision-making is not always linear and often influenced by factors that have not been clearly borne out of the literature.

Machine learning and AI can assist clinicians with this "art of medicine". Automating radiologic diagnoses, recommending appropriate treatment approaches, and predicting outcome following treatment all fall within the scope of AI. Further, when compared with our own abilities, AI can often perform as well if not better in terms of accuracy [4, $6,14-16,18,20]$. While the goal is not to replace the clinician, the possibility to improve our ability to provide optimal patient care represents an important one, and these developments should be seen as tools, which can complement clinical expertise and help us to master the information overflow.

\section{What is machine learning and artificial intelligence?}

Machine learning is a subset of AI, and both terms will be used interchangeably for the purposes of this editorial. These can be described as advanced statistical techniques that use computer algorithms to model complex relationships between variables. They are considered "advanced" because the relationships that are assessed can be more complex than with traditional statistics. In essence, the computer algorithms can "learn" from the data automatically, with minimal explicit and direct human computer programming.

Simply put, ML represents a new way to get from "input" to "output". Typically, this begins with a large dataset. The data are divided into "predictor" variables (such as age, sex, baseline subjective outcome scores, injury history, and image files) and "outcome" variables (such as subjective or objective failure, revision surgery, and particular diagnosis). Tasked with predicting a particular outcome of interest, the computer model analyses the relationship between each set of "predictor" variables and the specified outcome. The analysis can identify which factors are focal in predicting the outcome, quantify and rank them in order of importance, and develop an algorithm to predict the outcome. This learning phase of the algorithm is commonly referred to as "training" and is dependent on the quality of the dataset. When used in orthopaedics, these trained algorithms can then be applied to future patients.

Consider, for example, a study to predict revision surgery from a database containing information on 20,000 patients who underwent surgery. A standard ML approach would be to take 15,000 of these patients to "learn" from while saving the remaining 5,000 patients to test the accuracy of the resulting algorithm. The "outcome" variable in this case is revision surgery, while any other variable contained within the registry could be considered a "predictor". During the "learning" phase, the ML model would evaluate which predictor variables were associated with the revision surgery outcome, and how they interact with each other to affect the risk of a patient having a subsequent revision. The model can then estimate a numeric risk score for patients based on their unique predictor profile. After the model has "learned" what factors are associated with revision and how to quantify that risk, it can be tested on the 5,000 holdout patients (where the outcome is known) to determine the accuracy of the algorithm. Once the model has been trained and its accuracy established, it can then be re-trained on the entire dataset prior to implementation. The result is a validated algorithm that has considered complex interactions between the variables and that may yield more accurate, patient-specific, predictive capability. It is also possible for the algorithm to continue to improve over time, as it learns from new data it receives prospectively.

How the model "learns" from data and develops the algorithms used for outcome prediction as well as the pitfalls in these steps requires a longer discussion and is beyond the scope of this editorial. In general, the computer is programed to "learn" the way we do as humans. Pattern recognition makes up the basis of this approach. Just as years of textbooks, physical examinations, and clinical experience formulate the clinician's approach to their patient, a computer algorithm can be developed to do the same using many data points which mimic human experience. Once the ML model has seen enough "patients" and can link their unique characteristics to the outcome of interest (training phase), the model can begin to predict the outcome for other patients based on its experience. Additionally, ML models can learn from their mistakes or misclassifications, which can lead to improved accuracy over time, analogous to the surgeon progressing through residency and into independent practice.

\section{Recent examples in orthopaedic surgery and sports medicine}

There are a growing number of examples of ML application to orthopaedic conditions. Image-based analysis has led to the development of programs capable of diagnosing paediatric elbow fractures [4] and hip fractures [18, 20] — with accuracy similar to an orthopaedic surgeon. These programs may be beneficial for primary or urgent care settings to aid in accurate diagnosis and appropriate orthopaedic referral. Programs have also been developed to automate radiographic measurements such as coronal knee alignment [15] and acetabular component inclination 
and version [14]. Standardizing these measurements not only offers time savings in the clinical setting, but measurement consistency for future studies.

Recent studies have also applied ML for the purposes of orthopaedic outcome prediction and prognostication. Machine learning has been used to predict minimal clinically important difference (MCID) of patient-reported outcome (PRO) following osteochondral allograft transplantation for cartilage defects around the knee $[12,13]$. Similarly, MCID following hip arthroscopy has been evaluated using ML analysis, resulting in a tool that may guide decision-making regarding surgical outcomes and expectations [7]. Machine learning has also led to the development of algorithms capable of predicting the risk of knee arthritis progression following subchondral insufficiency fracture [11], the need for an overnight stay in the hospital following ACL reconstruction [9], or need for prolonged opioid use after elective knee arthroscopy [10].

Just as with orthopaedics, the field of sports medicine is experiencing similar growth in AI development and application. Early focus was on "analytics" and "advanced statistics" evaluating athlete performance and outcome prediction. Recently, more clinically relevant studies have emerged that focus on injury prediction [19]. These early efforts are encouraging and may lead to the development of comprehensive injury prevention initiatives founded in, or incorporating, AI.

\section{Unlocking the potential}

We are in a period of exponential growth regarding the use of AI across all specialties, including orthopaedic surgery, as clinicians and researchers alike gain comfort and proficiency with these novel techniques. As data collection improves, so too will the possibilities associated with AI which include more accurate diagnostics, patient-specific treatment approaches, improved outcome prediction, and augmented education. A basic understanding of what ML entails and how it can be used to impact orthopaedic surgery and patient care is essential for the surgeon of today, let alone tomorrow.

\section{Acknowledgements Not applicable.}

Author contributions All listed authors have contributed substantially to this work: RKM, AP, and CL performed primary manuscript preparation. AG, TT, and RS assisted with editing and final manuscript preparation. All authors read and approved the final manuscript.

Funding No funding was received.

\section{Declarations}

Conflict of interest The authors declare that they have no conflict of interest.

Ethical approval Not applicable.

Informed consent Not applicable.

\section{References}

1. Ardern CL, Ekås GR, Grindem H, Moksnes H, Anderson AF, Chotel F, Cohen M, Forssblad M, Ganley TJ, Feller JA, Karlsson J, Kocher MS, LaPrade RF, McNamee M, Mandelbaum B, Micheli L, Mohtadi N, Reider B, Roe J, Seil R, Siebold R, SilversGranelli HJ, Soligard T, Witvrouw E, Engebretsen L (2018) 2018 International Olympic Committee consensus statement on prevention, diagnosis and management of paediatric anterior cruciate ligament (ACL) injuries. Br J Sports Med 52:422-438

2. Berwick DM (2003) Disseminating innovations in health care. JAMA 289:1969-1975

3. Cabitza F, Locoro A, Banfi G (2018) Machine learning in orthopedics: a literature review. Front Bioeng Biotechnol 6:75

4. Choi JW, Cho YJ, Lee S, Lee J, Lee S, Choi YH, Cheon J-E, Ha JY (2020) Using a dual-input convolutional neural network for automated detection of pediatric supracondylar fracture on conventional radiography. Investig Radiol 55:101-110

5. Collins GS, Reitsma JB, Altman DG, Moons KGM (2015) Transparent reporting of a multivariable prediction model for individual prognosis or diagnosis (TRIPOD): the TRIPOD statement. Ann Intern Med 162:55-63

6. Esteva A, Kuprel B, Novoa RA, Ko J, Swetter SM, Blau HM, Thrun S (2017) Dermatologist-level classification of skin cancer with deep neural networks. Nature 542:115-118

7. Kunze KN, Polce EM, Clapp I, Nwachukwu BU, Chahla J, Nho SJ (2021) Machine learning algorithms predict functional improvement after hip arthroscopy for femoroacetabular impingement syndrome in athletes. J Bone Jt Surg 103:1055-1062

8. Liu Y, Chen P-HC, Krause J, Peng L (2019) How to read articles that use machine learning: users' guides to the medical literature. JAMA 322:1806-1816

9. Lu Y, Forlenza E, Cohn MR, Lavoie-Gagne O, Wilbur RR, Song BM, Krych AJ, Forsythe B (2020) Machine learning can reliably identify patients at risk of overnight hospital admission following anterior cruciate ligament reconstruction. Knee Surg Sports Traumatol Arthrosc. https://doi.org/10.1007/s00167-020-06321-w

10. Lu Y, Forlenza E, Wilbur RR, Lavoie-Gagne O, Fu MC, Yanke AB, Cole BJ, Verma N, Forsythe B (2021) Machine-learning model successfully predicts patients at risk for prolonged postoperative opioid use following elective knee arthroscopy. Knee Surg Sports Traumatol Arthrosc. https://doi.org/10.1007/ s00167-020-06421-7

11. Pareek A, Parkes CW, Bernard CD, Abdel MP, Saris DBF, Krych AJ (2020) The SIFK score: a validated predictive model for arthroplasty progression after subchondral insufficiency fractures of the knee. Knee Surg Sports Traumatol Arthrosc 28:3149-3155

12. Ramkumar PN, Karnuta JM, Haeberle HS, Owusu-Akyaw KA, Warner TS, Rodeo SA, Nwachukwu BU, Williams RJ (2021) Association between preoperative mental health and clinically meaningful outcomes after osteochondral allograft for cartilage defects of the knee: a machine learning analysis. Am J Sports Med 49:948-957 
13. Ramkumar PN, Karnuta JM, Haeberle HS, Rodeo SA, Nwachukwu BU, Williams RJ (2021) Effect of preoperative imaging and patient factors on clinically meaningful outcomes and quality of life after osteochondral allograft transplantation: a machine learning analysis of cartilage defects of the knee. Am J Sports Med 49:2177-2186

14. Rouzrokh P, Wyles CC, Philbrick KA, Ramazanian T, Weston AD, Cai JC, Taunton MJ, Lewallen DG, Berry DJ, Erickson BJ, Maradit Kremers H (2021) A deep learning tool for automated radiographic measurement of acetabular component inclination and version after total hip arthroplasty. J Arthroplasty 36(7):25102517.e6. https://doi.org/10.1016/j.arth.2021.02.026

15. Schock J, Truhn D, Abrar DB, Merhof D, Conrad S, Post M, Mittelstrass F, Kuhl C, Nebelung S (2021) Automated analysis of alignment in long-leg radiographs by using a fully automated support system based on artificial intelligence. Radiol Artif Intell 3:e200198

16. Senior AW, Evans R, Jumper J, Kirkpatrick J, Sifre L, Green T, Qin C, Žídek A, Nelson AWR, Bridgland A, Penedones H, Petersen S, Simonyan K, Crossan S, Kohli P, Jones DT, Silver D, Kavukcuoglu K, Hassabis D (2020) Improved protein structure prediction using potentials from deep learning. Nature 577:706-710

17. Subramanian P, Kantharuban S, Subramanian V, Willis-Owen SAG, Willis-Owen CA (2011) Orthopaedic surgeons: as strong as an ox and almost twice as clever? Multicentre prospective comparative study. BMJ 343:d7506-d7506

18. Urakawa T, Tanaka Y, Goto S, Matsuzawa H, Watanabe K, Endo N (2019) Detecting intertrochanteric hip fractures with orthopedist-level accuracy using a deep convolutional neural network. Skelet Radiol 48:239-244

19. Van Eetvelde H, Mendonça LD, Ley C, Seil R, Tischer T (2021) Machine learning methods in sport injury prediction and prevention: a systematic review. J Exp Orthop 8:27

20. Yamada Y, Maki S, Kishida S, Nagai H, Arima J, Yamakawa N, Iijima Y, Shiko Y, Kawasaki Y, Kotani T, Shiga Y, Inage K, Orita S, Eguchi Y, Takahashi H, Yamashita T, Minami S, Ohtori S (2020) Automated classification of hip fractures using deep convolutional neural networks with orthopedic surgeon-level accuracy: ensemble decision-making with antero-posterior and lateral radiographs. Acta Orthop 91:699-704

Publisher's Note Springer Nature remains neutral with regard to jurisdictional claims in published maps and institutional affiliations. 